\title{
Equivalent Circuit-Based Analysis of CMUT Cell Dynamics in Arrays
}

\author{
H. Kağan Oğuz, Student Member, IEEE, Abdullah Atalar, Fellow, IEEE, \\ and Hayrettin Köymen, Senior Member, IEEE
}

\begin{abstract}
Capacitive micromachined ultrasonic transducers (CMUTs) are usually composed of large arrays of closely packed cells. In this work, we use an equivalent circuit model to analyze CMUT arrays with multiple cells. We study the effects of mutual acoustic interactions through the immersion medium caused by the pressure field generated by each cell acting upon the others. To do this, all the cells in the array are coupled through a radiation impedance matrix at their acoustic terminals. An accurate approximation for the mutual radiation impedance is defined between two circular cells, which can be used in large arrays to reduce computational complexity. Hence, a performance analysis of CMUT arrays can be accurately done with a circuit simulator. By using the proposed model, one can very rapidly obtain the linear frequency and nonlinear transient responses of arrays with an arbitrary number of CMUT cells. We performed several finite element method (FEM) simulations for arrays with small numbers of cells and showed that the results are very similar to those obtained by the equivalent circuit model.
\end{abstract}

\section{INTRODUCTION}

$\mathrm{T}$ HE primary purpose of transducer arrays used in many ultrasound applications is to radiate powerful, beamformed acoustic signals. The acoustic crosstalk that occurs between the closely packed cells of capacitive micromachined ultrasonic transducer (CMUT) arrays is considered important, because it impairs both beamforming and powerful radiation. The effect of crosstalk had been assessed extensively by experiments and measurements. It is hypothesized that the crosstalk is caused by either of two phenomena. One phenomenon is the waves propagating in the silicon substrate [1]-[3]. The other phenomenon is a result of the acoustic interactions that occur when the sound pressure fields of the transducers exert force on each other through the immersion medium. This phenomenon has been recognized in sonar transducer arrays for many decades, and its significant effects on array performance have been studied by means of the mutual radiation impedance between the transducers [4]-[6]. In this work, we are concerned only with the second crosstalk mechanism, which has an evident effect on CMUT arrays [7]-[10], and ignore the first one.

CMUT membranes have low mechanical impedance, which makes them inherently suitable for immersion applications. With this major advantage, CMUTs are ca-

Manuscript received November 20, 2012; accepted February 14, 2013. The authors are with the Electrical and Electronics Engineering Department, Bilkent University, Ankara, Turkey (e-mail: oguz@ee.bilkent. edu.tr).

DOI http://dx.doi.org/10.1109/TUFFC.2013.2660 pable of transmitting and receiving wideband acoustic signals. On the other hand, a low mechanical impedance means low quality factor (Q) of mechanical resonance. It brings with it severe effects resulting from mutual acoustic interactions, which are manifested in the operational bandwidth of the transducer [8], [9], [11]. As is the case for other electromechanical transducer types, this drawback can cause degradation in acoustic power radiation [12]-[14], distortion in sound beam patterns [4] and sometimes even failure of the electronic amplifiers that drive the transducers [15].

Surface micromachining technology has made possible batch fabrication of large CMUT arrays, offering the opportunity to integrate driving electronics. Depending on the precision of the manufacturing process, array designers may be presented with a great number of different configurations to attempt. However, the lack of appropriate design and analysis tools prevents investigation of the mutual acoustic coupling effects occurring in large arrays.

Finite element method (FEM) tools are widely used in the analysis of acoustic transducers. Although its predictions are very accurate, the analysis process is very time consuming and thus generally impractical to use for design purposes. It is not feasible to analyze large arrays without making some simplifying assumptions in the FEM model. There exist reduced FEM models that are used to simulate long 1-D CMUT arrays [1], [11], [16], [17]. In these models, the array structure is assumed to be infinitely long, so that only a single periodic portion is modeled to be electrically driven in phase with the rest of the cells. However, these simple reduced FEM models are not exact, and the important effects of acoustic interactions cannot be accurately investigated [11]. Therefore, FEM is not suitable for use in designing CMUT arrays, although it can be employed to verify a particular design with a small number of cells.

It is common to use Mason's linear electrical equivalent circuit when analyzing single as well as multiple CMUT cells. Once the self- and mutual-radiation impedances of the cells are known and taken into account, an equivalent circuit for an immersed CMUT array can be built [8], [9], [18]. However, the predictions of this equivalent model are not satisfactory [8], [19].

Recently, we developed an accurate nonlinear equivalent circuit model for CMUT cells in immersion with partial or full electrodes [19]. The model is valid in the presence of any static external force and can be applied in either transmit or receive modes as long as the membrane does not touch the substrate. In this paper, we use 
this model as a building block for CMUT arrays. The equivalent circuit including all cells in the array is coupled at their acoustic terminals through an impedance matrix. The matrix contains the self-radiation impedance of each cell and the mutual radiation impedance between every pair of cells. We present an accurate and easy-to-compute approximation for the mutual radiation impedance derived by Porter [20]. The approximate expression can be applied to large arrays. Employing the proposed model, we discuss some aspects of mutual acoustic interactions in CMUT cell clusters and elements. Where possible, we perform FEM simulations and show that the results obtained by the equivalent circuit model and FEM are very consistent.

\section{An Approximation for the Mutual Radiation IMPEDANCE BETWEEN CMUTS}

CMUT array elements consist of multiple cells, which are usually closely packed and electrically driven in parallel. A generic CMUT array is shown in Fig. 1, where $a$ is the radius of the cells, $d_{i j}$ is the center-to-center separation between any two cells, and $M$ and $K$ denote the number of cells in the rows and columns. The total radiation impedance of the $i$ th cell is defined as

$$
Z_{i}=Z_{i i}+\sum_{\substack{j=1 \\ i \neq j}}^{N} \frac{v_{j}}{v_{i}} Z_{i j},
$$

where $N=M K$ is the number of cells, $Z_{i i}$ is the selfradiation impedance of the $i$ th cell when it is located on an infinite rigid plane baffle, $v_{i}$ and $v_{j}$ are the reference velocities for the $i$ th and $j$ th cells, and $Z_{i j}$ is the mutual radiation impedance between them [15]. For a given pair of cells, the value of $Z_{i j}$ depends only on the radius of each cell and the separation between them normalized with the wavelength in the immersion medium.

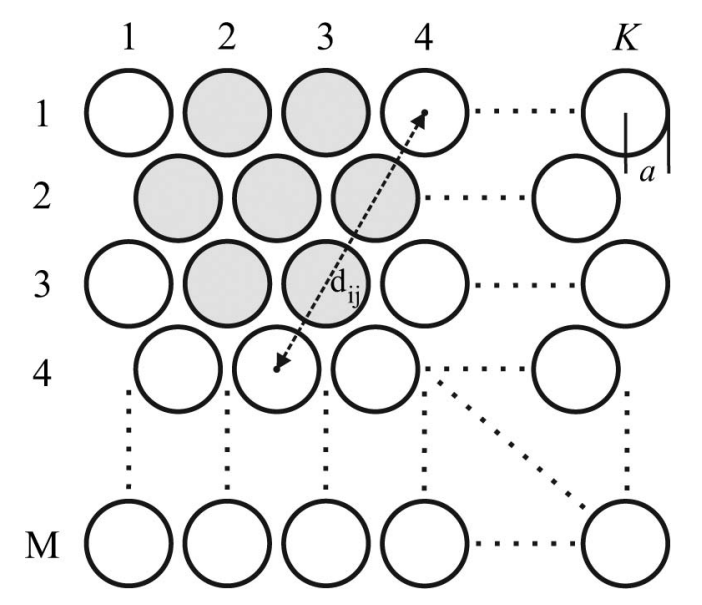

Fig. 1. Configuration of a rectangular array of CMUT cells, where the center-to-center displacement between the $i$ th and $j$ th cell is denoted.

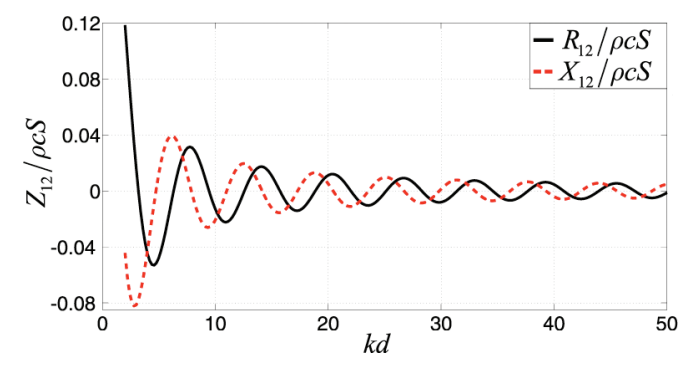

Fig. 2. Mutual radiation resistance, $R_{12}$, and reactance, $X_{12}$, between two clamped circular radiators normalized to $\rho c S$ when $k a=1 . \rho$ and $c$ are the density and velocity, respectively, of sound in the immersion medium, and $S=\pi a^{2}$ is the surface area of each radiator. The impedance values are referred to spatial rms velocity.

The acoustic force at the radiation interface of each cell can be interpreted in matrix form with

$$
\left[\begin{array}{c}
F_{1} \\
F_{2} \\
\vdots \\
F_{N}
\end{array}\right]=\left[\begin{array}{cccc}
Z_{11} & Z_{12} & \cdots & Z_{1 N} \\
Z_{21} & Z_{22} & \cdots & Z_{2 N} \\
\vdots & \vdots & \ddots & \vdots \\
Z_{N 1} & Z_{N 2} & \cdots & Z_{N N}
\end{array}\right]\left[\begin{array}{c}
v_{1} \\
v_{2} \\
\vdots \\
v_{N}
\end{array}\right],
$$

where $F_{i}$ and $v_{i}$ represent the rms force and the rms velocity of the individual cells, respectively. The square matrix, $\mathbf{Z}=\left[Z_{i j}\right]$, is the impedance matrix. If all the transducers in the array are identical, the self-radiation impedance is the same for all of them. According to the acoustical reciprocity theorem, $Z_{j i}=Z_{i j}$, so that $\mathbf{Z}$ is a complex symmetric matrix.

Porter studied the self- and mutual-radiation impedances of flexural disks with different boundary conditions, located on an infinite rigid plane baffle [20]. Infinite series expressions for the real and imaginary parts of the mutual radiation impedance, $Z_{12}$, between two clamped radiators are given in [20, Eqs. 44 and 46]. ${ }^{1}$ For $k a=1$, Fig. 2 shows the variation of $Z_{12}$ as a function of $k d$, where $k$ is the wavenumber in the immersion medium and $d$ is the center-to-center distance between the two circular radiators. In this work, the impedance values are referred to the spatial $\mathrm{rms}$ velocity of the radiator [19]. These values are $5 / 9$ times the values obtained by Porter [20], where the average velocity rather than rms velocity is chosen as the reference velocity.

$Z_{12}$ is an inseparable expression of $k a$ and $k d$. By its nature, it is a slowly decaying function of $k d$ [20]. This implies that for a large CMUT array, the combined interactions from distant cells may become highly effective on the acoustic load impedance experienced by each cell. For this reason, the mutual radiation impedance between all pairs of cells needs to be taken into account, which may introduce a huge $\mathbf{Z}$ matrix to compute.

If we carefully analyze the real and imaginary parts of $Z_{12}$, we see that both decays proportionally with $k d$ and the phase difference between them is always nearly $90^{\circ}$.

\footnotetext{
${ }^{1}$ The factor $1 / i$ is missing on the right-hand side of Eq. 46 in [20].
} 
This convinces us to obtain an accurate approximation of the following form:

$$
\frac{Z_{12}}{\rho c S} \cong A(k a) \frac{\sin (k d)+j \cos (k d)}{k d}, \text { for } k a<5.5,
$$

where $A(k a)$ is found by curve fitting and it is a complex function as depicted in Fig. 3. To obtain the real and imaginary parts of $A(k a)$, tenth-order polynomials are used, the coefficients of which are given in the Appendix. When $k a \ll 1$ and $k a \ll k d, A(k a)=(5 / 9)(k a)^{2} / 2[20]$. To calculate $Z_{i j}$ in (2), $d$ in (3) must be replaced with the corresponding $d_{i j}$.

With (3), the dependence of $Z_{12}$ on $k a$ and $k d$ is now separated. For $k a>5.5$, this approximation is not correct, because in the vicinity of $k a=2 \pi$ the decay of $Z_{12}$ is not simply proportional to $k d$. However, beyond this limit the values of $Z_{12}$ are very small compared with the selfradiation resistance and can be ignored. ${ }^{2}$

\section{An Equivalent Circuit for CMUT Arrays}

A CMUT array can be built by combining several of the equivalent circuits developed in [19], through the appropriate $\mathbf{Z}$ matrix, as demonstrated in Fig. 4. The equivalent circuit model and the mutual radiation impedance described in Section II are consistent, because both are obtained for circular disks with a clamped edge. There are two important, albeit ordinary assumptions for this model. First, the array is located on an infinite, rigid plane baffle, so that acoustic radiation takes place in the semiinfinite fluid domain [20]. Second, higher order modes of the cells in the array are assumed not to be excited in the frequency region of interest [14].

\section{Acoustic Interactions Between Closely Packed CMUT Cells}

In this section, we study the impact of acoustic interactions in different CMUT array elements when all of the cells are biased and driven in parallel with a $1 \mathrm{~V}$ peak ac voltage. The cells are closely packed, with the edgeto-edge separation between them being $a / 10$. A commercial circuit simulator (Advanced Design System, Agilent Technologies, Palo Alto, CA) is used to obtain the linear and nonlinear responses of the equivalent circuit model of CMUT elements with arbitrary number of cells. The effects of cell apertures and their relative locations in the elements are examined. The results are compared with 3-D FEM results (Ansys, Ansys Inc., Canonsburg, PA) and shown to be similar to the equivalent model predic-

\footnotetext{
${ }^{2}$ For example, at $k a=2 \pi$, when $k d=4 \pi, R_{12} / \rho c S=4.32 \times 10^{-4}$, but when $k d=16 \pi$, it decays rapidly and the oscillation amplitude of $R_{12} /$ $\rho c S$ becomes approximately $7 \times 10^{-6}$.
}

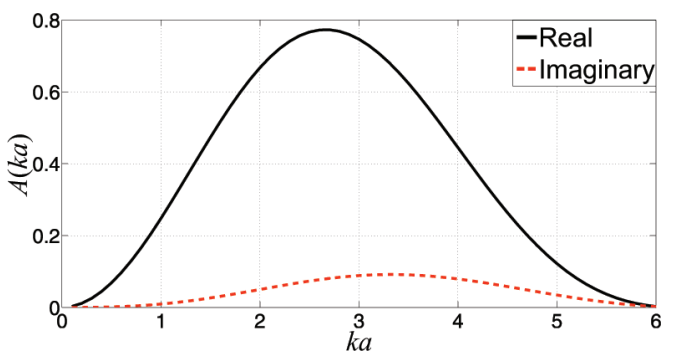

Fig. 3. The real and imaginary parts of the $k a$-dependent term, $A(k a)$, of the approximate mutual radiation impedance expression given in (3) for $k a<5.5$.

tions. The circuit simulations are completed within a few seconds, whereas FEM simulations take a few hours.

\section{A. Effects of Cell Aperture and Location}

Three CMUT cells with different radii and thicknesses that resonate at around $3.5 \mathrm{MHz}$ in water are considered. They are designated CMUTs I, II, and III, with $a \simeq \lambda / 4$, $\lambda / 8$, and $\lambda / 16$, respectively, where $\lambda$ is the wavelength in water at $3.5 \mathrm{MHz}$. The physical properties of these CMUTs and the dc bias voltages applied to them are given in Table I. Notice that cells with larger radii have thicker membranes, resulting in the same resonance frequency.

We investigate the effects of mutual interactions between the cells for 2-cell, 3-cell, and 4-cell elements (Fig. $5)$. Note that for 2-cell and 3-cell elements, cell locations are symmetric. Thus, there are two different values in the $\mathbf{Z}$ matrix: the self-radiation impedance, $Z_{11}$, and the mutual radiation impedance, $Z_{12}$. For the 4 -cell case, there is another term, $Z_{14}$, because of the dissimilar distance between the diagonal cells.

Equivalent circuit simulations are done for CMUTs I, II, and III to observe the effects of cell radius on the mutual acoustic coupling that takes place in each particular cell configuration. The total electrical conductances, $G$, of the parallel connected cells for all cases are plotted in Fig.

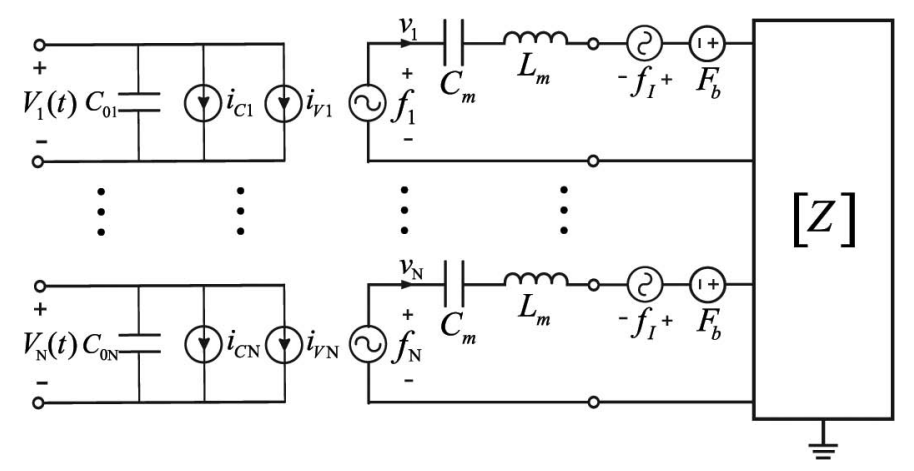

Fig. 4. Equivalent circuit representation of an array of $N$ CMUTs. $F_{b}$ is the static external force, such as that caused by atmospheric pressure. $f_{I}$ is the dynamic external force, such as that caused by an incident acoustic signal. For thick membranes with $a / t_{m}<10$, a correction for the membrane compliance, $C_{m}[24]$, is used. 


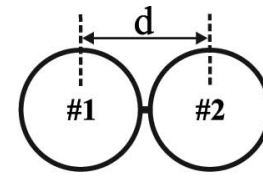

(a)

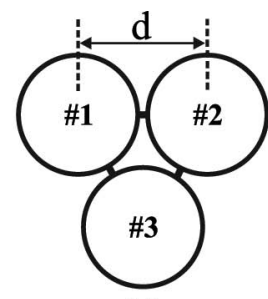

(b)

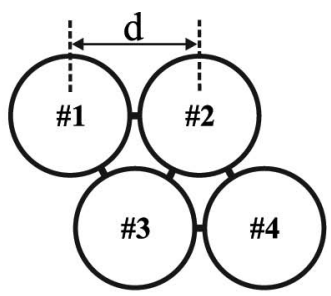

(c)
Fig. 5. Configuration of CMUT array elements with (a) two, (b) three, and (c) four closely packed cells, located on an infinite rigid baffle.

6. In each graph, the values for a single cell placed on an infinite rigid baffle are also given for reference. According to the results, each type of CMUT appears to be influenced by the mutual interactions, but in a different manner. Calculated for $a \simeq \lambda / 4$, Fig. 6 (a) shows that there is a noticeable shift in the resonance frequency to higher values as the number of cells increases. Fig. 6(b) and Fig. $6(\mathrm{c})$ show that there is a frequency shift to lower values.

There is a prominent effect of mutual acoustic coupling for the 4-cell configuration. For $a \simeq \lambda / 8$ and $\lambda / 16$, conductance curves indicate the presence of spurious resonances. It is clear that these resonances are caused by the acoustic coupling of cells through the medium and this phenomenon is accurately modeled by using the mutual impedances. Note that the same effect also exists for $a \simeq \lambda / 4$, but is quite subtle. To further investigate this anomaly, we observed the magnitude of the peak displacement, $x_{\mathrm{p}}$, of the cells. The results with respect to frequency are shown in Fig. 7. The behavior of the cells in the same row is substantially different regardless of the cell apertures, because they experience different acoustic loads from the immersion medium. However, the results indicate that this resonance effect is more pronounced when the cell radius is small.

\section{B. 6-Cell and 7-Cell Elements}

In this section, hexagonally arranged CMUT array elements are discussed. The geometries of two elements with six and seven cells are shown in Fig. 8. The total radiation impedance experienced by the cell in the middle of

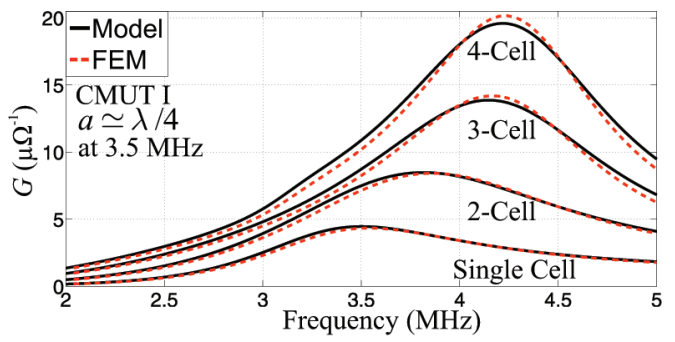

(a)

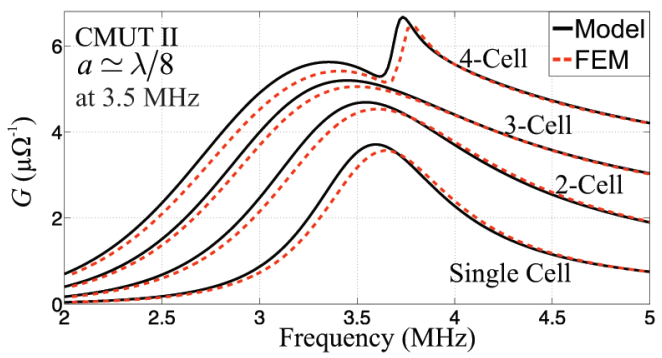

(b)

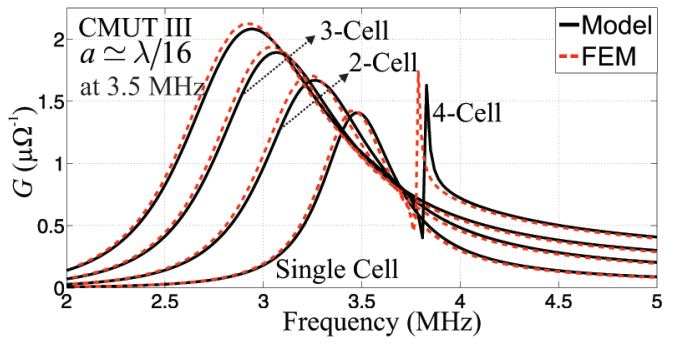

(c)

Fig. 6. The electrical conductance of a single cell and 2-cell, 3-cell, and 4-cell cases using CMUTs (a) I, (b) II, and (c) III for each cell. The cells are located on an infinite rigid plane baffle and they are immersed in water.

the 7-cell element will not be the same as that for those located at the sides. The effect of this difference on element behavior is explored for different cell radii (CMUTs I, II, and III).

The electrical conductances of each array, where all the cells are biased and driven electrically in parallel, are plotted in the graphs in the upper row of Fig. 9, for different cell radii. It is seen that the presence of the center cell significantly alters the conductance, and its effect becomes

TABle I. Dimensions and Bias Voltages of the CMUT Cells Used in the Simulations.

\begin{tabular}{|c|c|c|c|}
\hline Parameter & $\begin{array}{l}\text { CMUT I } \\
(a \simeq \lambda / 4)\end{array}$ & $\begin{array}{l}\text { CMUT II } \\
(a \simeq \lambda / 8)\end{array}$ & $\begin{array}{l}\text { CMUT III } \\
(a \simeq \lambda / 16)\end{array}$ \\
\hline Membrane radius, $a(\mu \mathrm{m})$ & 104 & 53 & 26.9 \\
\hline Membrane thickness, $t_{\mathrm{m}}(\mu \mathrm{m})$ & 13 & 4.3 & 1.3 \\
\hline Gap height, $t_{\mathrm{ga}}(\mathrm{nm})$ & 150 & 200 & 270 \\
\hline Young's modulus, $Y_{0}(\mathrm{GPa})$ & 320 & 320 & 320 \\
\hline Density, $\rho\left(\mathrm{g} / \mathrm{cm}^{3}\right)$ & 3.27 & 3.27 & 3.27 \\
\hline Poisson's ratio, $\sigma$ & 0.263 & 0.263 & 0.263 \\
\hline Insulator thickness, $t_{\mathrm{i}}(\mathrm{nm})$ & 100 & 100 & 100 \\
\hline Insulator permittivity, $\varepsilon_{r}$ & 4 & 4 & 4 \\
\hline Collapse voltage, $V_{\mathrm{c}}(\mathrm{V})$ & 90 & 97 & 100 \\
\hline Bias voltage, $V_{\mathrm{DC}}$ & $0.5 V_{\mathrm{c}}$ & $0.7 V_{\mathrm{c}}$ & $0.7 V_{\mathrm{c}}$ \\
\hline
\end{tabular}

$\lambda$ is the wavelength in water at $3.5 \mathrm{MHz}$. 


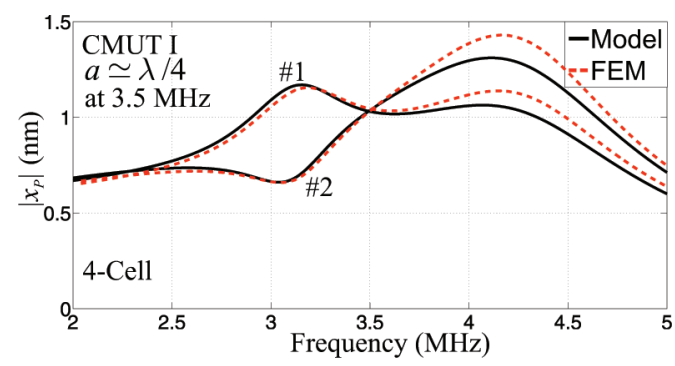

(a)

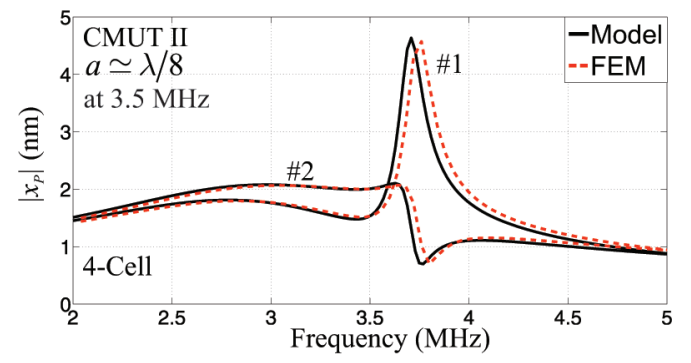

(b)

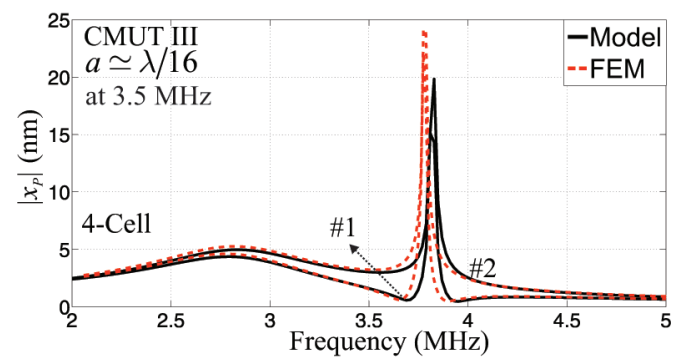

(c)

Fig. 7. The magnitude of the peak displacement of the cells in the first row of the 4-cell element using CMUTs (a) I, (b) II, and (c) III for each cell. The cells are located on an infinite rigid plane baffle and they are immersed in water.

quite dramatic as the aperture size decreases. The remaining graphs of Fig. 9 depict the magnitude and phase of the peak displacement of each cell. The anomalies in the conductance values are more clear when the displacements of the center cell and the edge cells differ the most. Note that for $a \simeq \lambda / 4$, the displacement of the center cell decreases at around $3 \mathrm{MHz}$. Around this frequency, the responses of the 6-cell and 7-cell elements are very similar, as indicated by their conductances. This is because the center cell of the 7-cell element acts virtually as a rigid plane, which is indeed the case for the 6 -cell array. But for $a \simeq \lambda / 16$, the cells at the edges of the 7 -cell element do not vibrate effectively at around $3.7 \mathrm{MHz}$. However, for $a \simeq \lambda / 8$, neither the peak displacement of the center cell nor that of the edge cells vanishes at the given frequency range.

It may be suspected that an introduction of loss in the electrical side may kill the spurious resonances. But, that did not occur. For example, excitation of the cells by a $50-\Omega$ resistive source or addition of a $0.1-\Omega$ resistance in series with each cell did not make a significant difference in the results.

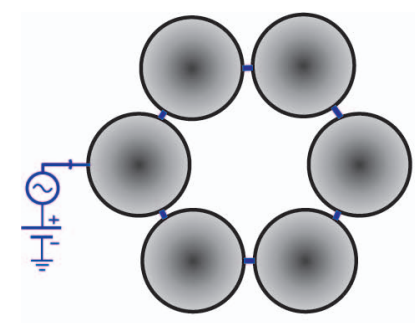

(a)

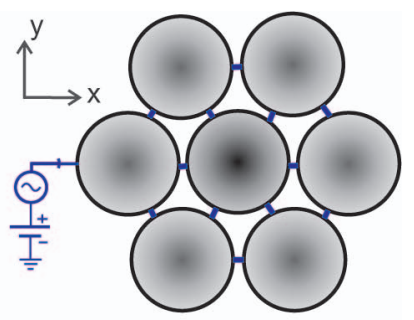

(b)
Fig. 8. (a) 6-cell and (b) 7-cell hexagonal CMUT array element geometries.

\section{1-D CMUT ARray ElEMENT}

Ordinarily, a 1-D CMUT array element has a length between $L=10 \lambda$ to $20 \lambda$ and a width of $W=\lambda / 2$, at the center frequency. It may contain 2 to 8 side-by-side cells in the width and, hence, may involve hundreds of cells in the whole array. It is impractical to build and simulate such an array with FEM. Therefore, reduced FEM models are usually utilized, in which 1-D CMUT array elements are assumed to be infinitely long [1], [16], [17]. In a reduced FEM model, only the smallest periodic portion of the element is modeled and the obtained results are assumed to be identical throughout the entire element. This method is misleading, because the mutual coupling effects for the infinitely long and finite elements are not the same [11]. In [21], we analyzed two $\lambda / 2$-width array elements using CMUT II cells, one with $L=10 \lambda$ and the other $L=20 \lambda$, and observed that the frequency response of the cells differ significantly compared with the FEM analysis performed with this assumption.

\section{A. Frequency Response Under Linear Conditions}

We modeled two elements with CMUT I and CMUT III cells with $L=10 \lambda$ and $W=\lambda / 2$ at $3.5 \mathrm{MHz}$ in water. The cells in the elements are placed in the same configuration given in Fig. 1, where for the one built with CMUT I cells, $M=22$ and $K=1$ and for the one built with CMUT III cells, $M=88$ and $K=4$. The edge-to-edge separation between each pair of cells is $a / 10$.

These elements are analyzed by the equivalent circuit model and the total electrical conductance, $G$, of each are as shown in Fig. 10 and in Fig. 11(a), respectively. It is important to note that the displacements obtained by the circuit model are different for each cell. This effect is very significant within the element built of CMUT III cells, for which many spurious modes are detected, as shown in Fig. 11(a).

The pressure field produced by $N$ cells that are located on an infinite rigid plane baffle is

$$
p(r, \theta, \phi)=j \frac{\rho c k S}{2 \pi} D(\theta) \sum_{i=1}^{N} U_{A_{i}} \frac{e^{-j k r_{i}}}{r_{i}},
$$


CMUT I $(a \simeq \lambda / 4$ at $3.5 \mathrm{MHz})$

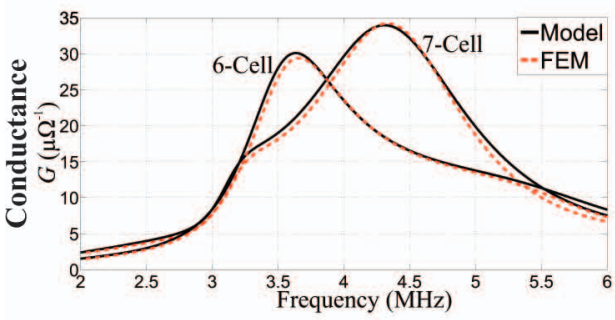

(a)

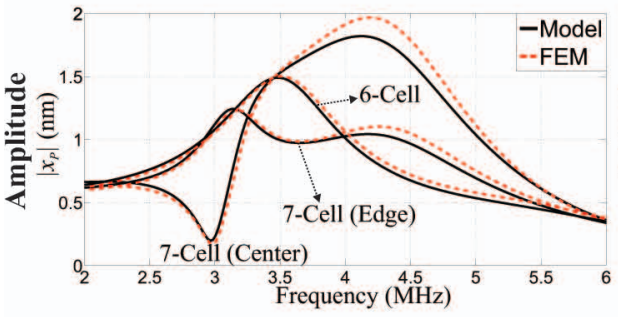

(b)

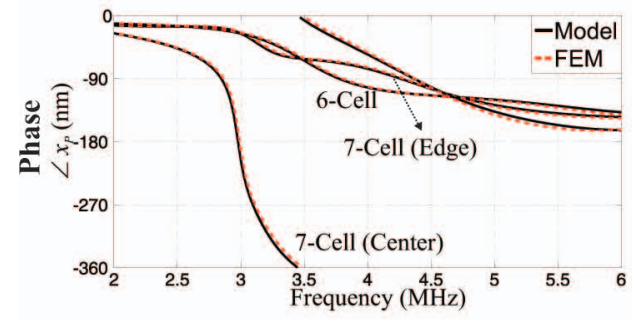

(c)
CMUT II $(a \simeq \lambda / 8$ at $3.5 \mathrm{MHz})$

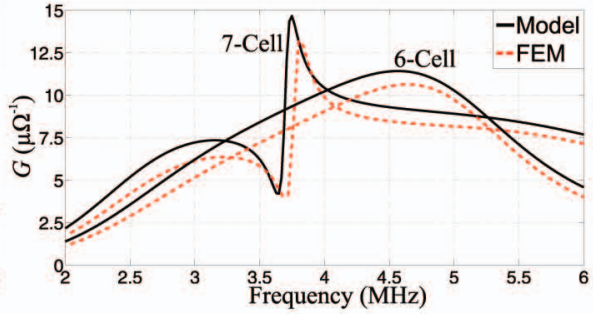

(d)

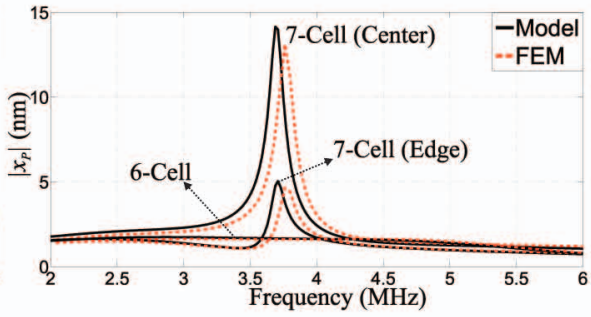

(e)

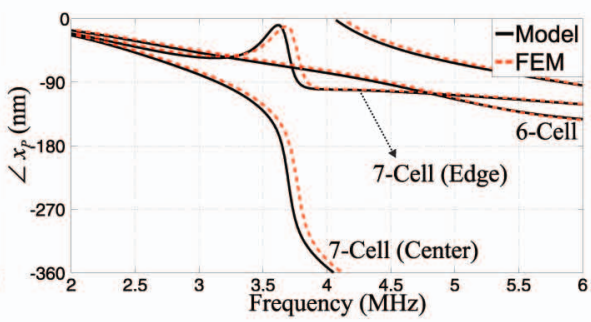

(f)
CMUT III $(a \simeq \lambda / 16$ at $3.5 \mathrm{MHz})$

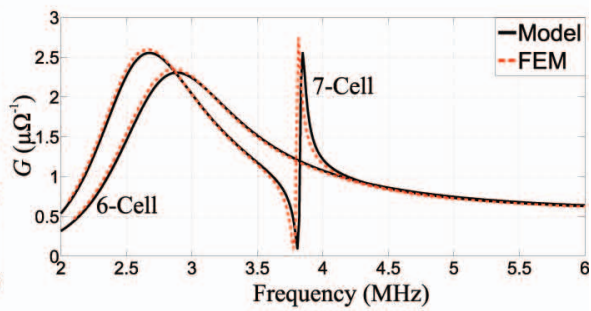

(g)

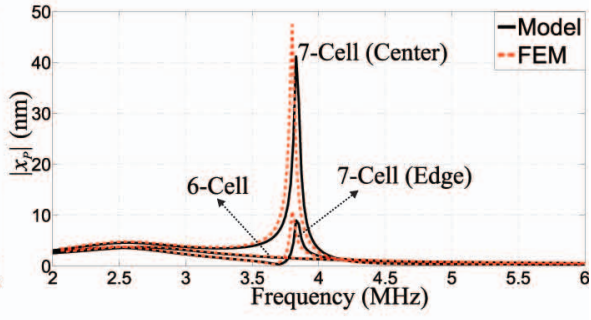

(h)

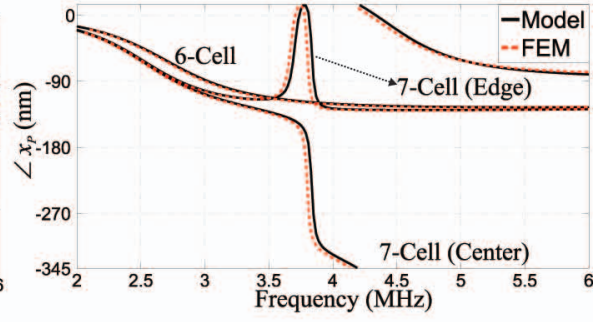

(i)

Fig. 9. The total electrical conductance of 6-cell and 7-cell hexagonal elements, and the amplitude and phase of the peak displacement, $x_{\mathrm{p}}$, of each cell in the elements built using CMUT I, II, and III cells. The cells are located on an infinite rigid plane baffle and they are immersed in water.

where $D(\theta)=48 J_{3}(k a \sin (\theta)) /(k a \sin (\theta))^{3}$ is the amplitude directivity function of the clamped edge radiator [20], $U_{A_{i}}$ is the average velocity phasor at the surface of the $i$ th cell, and $r_{i}$ is the radial distance of the $i$ th cell to the observation point in the medium.

Fig. 11(b) depicts the far-field radiation patterns and the transmitting voltage responses (TVRs) at 2.96 and $3 \mathrm{MHz}$ are given for the $10 \lambda$-long element built using CMUT III cells. At these frequencies, the corresponding displacement profiles of the element are given in Fig. 11(c) and Fig. 11(d), where two different mode shapes can be observed. At each frequency, both the magnitude and the

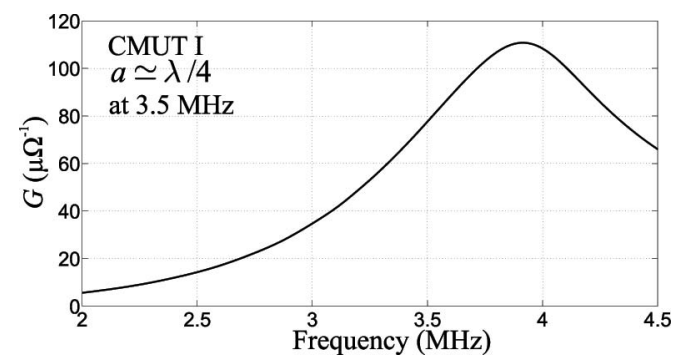

Fig. 10. The total electrical conductance of the 1-D CMUT array element, which consists of CMUT I cells, with $M=22$ and $K=1(L=10 \lambda$ and $W=\lambda / 2)$. phase of the cells are significantly different. The conductance variation in Fig. 11(a) indicates the existence of a spurious mode at $3 \mathrm{MHz}$, whereas $2.96 \mathrm{MHz}$ corresponds to a frequency point between two spurious modes. This behavior enhances the displacement amplitudes of the cells at $3 \mathrm{MHz}$, but reduces the main lobe of the radiation pattern while increasing the side lobe levels, with respect to $2.96 \mathrm{MHz}$.

For the $10 \lambda$-long element, the clarity of the spurious modes becomes less pronounced as the cell radii increase. Many spurious modes still exist when the elements consist of CMUT II $(a \simeq \lambda / 8)$ cells [21]. However, with CMUT I $(a \simeq \lambda / 4)$ cells with thicker membranes, the cell displacements are fairly uniform in the frequency band. Fig. 10 depicts the conductance of this element, which is free of any spurious mode.

\section{B. Transient Response}

By using the equivalent circuit model, the transient responses of $10 \lambda$-long elements are analyzed when they are excited by a square voltage pulse with 100 ns duration. The rise and fall times of the pulse are $10 \mathrm{~ns}$. The analyses done here are for the elements built using CMUT I and III cells, but in [21], the same analysis was also carried out for an element consisting of CMUT II cells. 


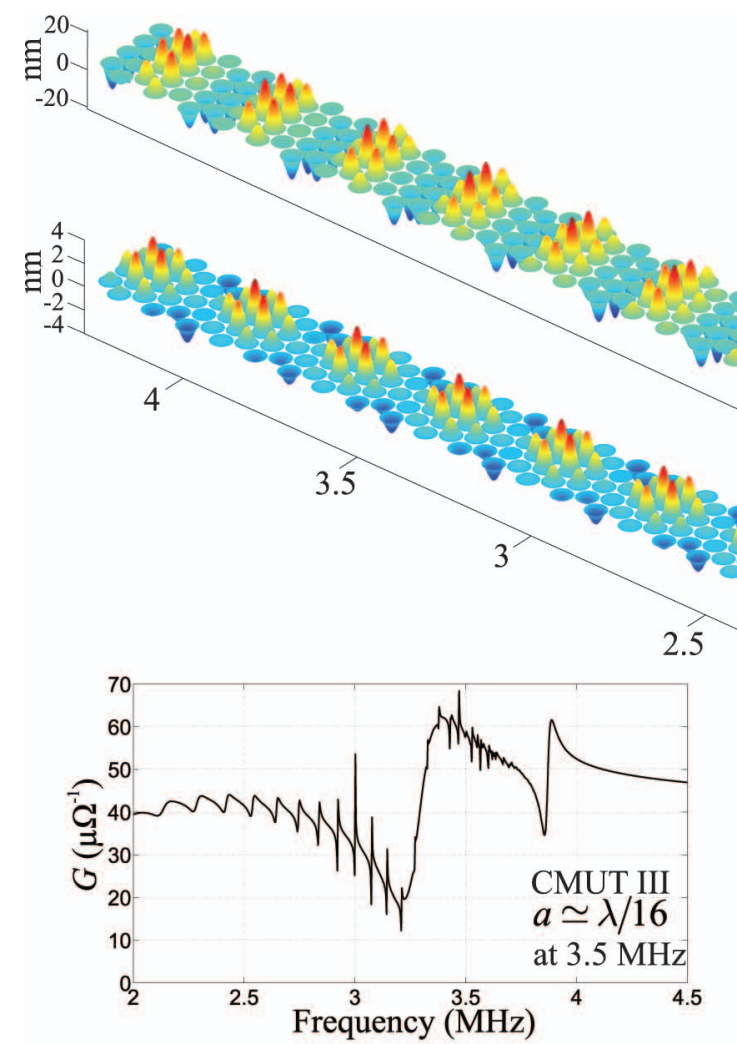

(a)

Fig. 11. (a) The total electrical conductance of the 1-D CMUT array element, which consists of CMUT III cells, with $M=88$ and $K=4(L=10 \lambda$ and $W=\lambda / 2$ ). (b) Far-field radiation patterns of the element at 2.96 and $3 \mathrm{MHz}$, where (c) and (d) show the corresponding displacement profiles, respectively.

For the CMUT I and CMUT III cells, the voltage is initially kept at $45 \mathrm{~V}$ and $70 \mathrm{~V}$, and then increased to $65 \mathrm{~V}$ and $90 \mathrm{~V}$ within the pulse duration, respectively. Figs. 12(a) and 13(a) show the time domain pressure pulses generated $1 \mathrm{~mm}$ away from the center of the elements. The frequency spectrum of these pressure pulses are given in Figs. 12(b) and 13(b). Note the presence of oscillations in Fig. 13(a), centered around $3.4 \mathrm{MHz}$, long after the pulse is applied. These are due to the existence of the spurious modes observed for the element built using CMUT III cells. The simulations show the existence of spurious modes clearly. They cause oscillations in the impulse response. Although it causes a little reduction in the amplitude of the focused beam, it results in an increase in the side lobe amplitude as well as an extension of impulse response duration.

\section{Conclusions}

We developed an equivalent circuit model for CMUT arrays, which can be easily employed in circuit simulators. We have shown that by using the proposed model, the effects of the mutual acoustic interactions can be analyzed very rapidly with an accuracy comparable to FEM. We provided an approximate expression for the mutual radiation impedance, which indicates its separability as a function of $k a$ and $k d$. We referred different array configurations and compared them for three different cell radii. The examples show that the design of a single cell and the

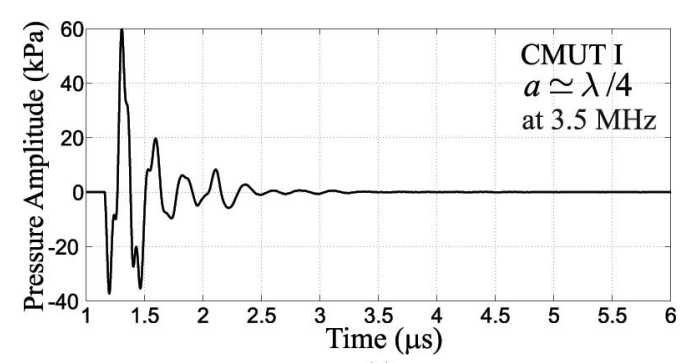

(a)

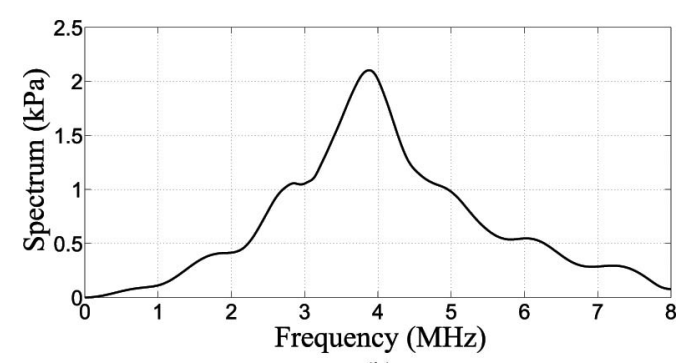

(b)

Fig. 12. (a) The pressure pulse generated at $1 \mathrm{~mm}$ away from the center of the $10 \lambda$-long element that is built of CMUT I cells, and (b) its spectrum. The element is located on an infinite rigid plane baffle and it is excited by a square voltage pulse with $0.1 \mu$ s duration. 


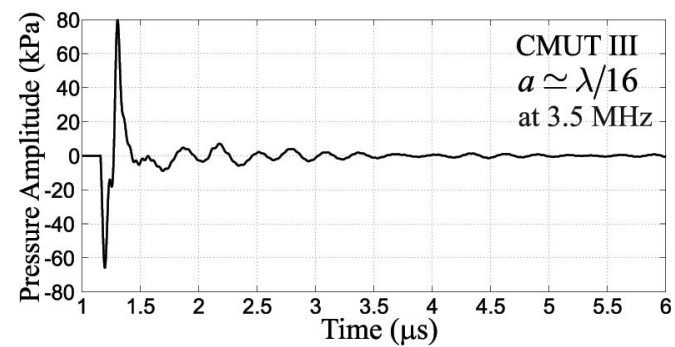

(a)

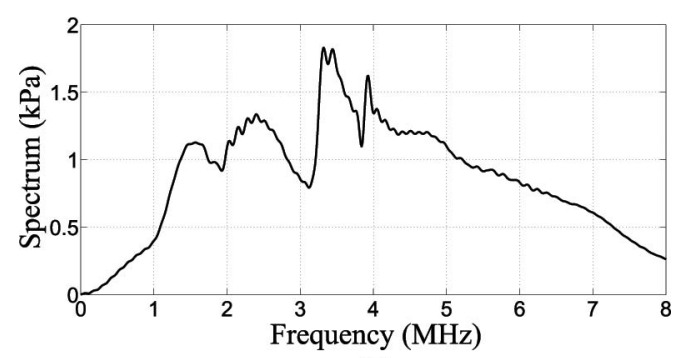

(b)

Fig. 13. (a) The pressure pulse generated at $1 \mathrm{~mm}$ away from the center of the $10 \lambda$-long element that is built of CMUT III cells, and (b) its spectrum. The element is located on an infinite rigid plane baffle and it is excited by a square voltage pulse with $0.1 \mu$ s duration.

design of an array cannot be considered separately. However, one can utilize the equivalent circuit model to design and optimize CMUT arrays.

The model lends itself readily to theoretical circuit analysis [22]. The electrical termination of the cells can easily be incorporated to examine its effect on the array performance. Also, the acoustic termination on nonradiating side of the cell, the backing, can be easily included as an appropriate impedance across the controlled force source, $f_{i}$, in the model. As future work, we plan to investigate the effect of mechanical Q of the cells on crosstalk, as well as the effects of acoustic backing and different electrical terminations, both theoretically and by simulation.

The employed circuit simulator must be capable of using frequency domain impedance data. Our circuit simulator can handle arrays with up to about ten thousand cells reasonably well. The number of cells in arrays realized by current design approaches can be quite high. For example, the linear array reported in [23] has more than 121000

TABle II. Polynomial Coefficients of Function $A(k a)$.

\begin{tabular}{lrr}
\hline & \multicolumn{1}{c}{ Real part } & Imaginary part \\
\hline$p_{10}$ & $-5.34237 \mathrm{e}-7$ & $-2.15428 \mathrm{e}-7$ \\
$p_{9}$ & $9.60487 \mathrm{e}-6$ & $6.64439 \mathrm{e}-6$ \\
$p_{8}$ & $-1.76278 \mathrm{e}-5$ & $-8.03963 \mathrm{e}-5$ \\
$p_{7}$ & $-6.79795 \mathrm{e}-4$ & $4.61167 \mathrm{e}-4$ \\
$p_{6}$ & $5.09681 \mathrm{e}-3$ & $-1.14002 \mathrm{e}-3$ \\
$p_{5}$ & $-7.47336 \mathrm{e}-3$ & $7.16523 \mathrm{e}-4$ \\
$p_{4}$ & $-2.50825 \mathrm{e}-2$ & $-2.72073 \mathrm{e}-3$ \\
$p_{3}$ & $-9.71827 \mathrm{e}-3$ & $1.47231 \mathrm{e}-2$ \\
$p_{2}$ & $2.87292 \mathrm{e}-1$ & $-9.01486 \mathrm{e}-4$ \\
$p_{1}$ & $-1.09336 \mathrm{e}-3$ & $1.92266 \mathrm{e}-4$ \\
$p_{0}$ & $8.62694 \mathrm{e}-5$ & $-1.42685 \mathrm{e}-5$ \\
\hline
\end{tabular}

cells in 192 elements. To analyze or design such an array, the model must be solved with a circuit simulator capable of handling very large circuits.

\section{APPENDIX}

The function $A(k a)$ can be calculated with the tenthorder polynomial,

$$
A(k a)=\sum_{n=0}^{10} p_{n}(k a)^{n}
$$

where the real and imaginary parts of its complex coefficients are given in Table II.

\section{REFERENCES}

[1] B. Bayram, M. Kupnik, G. G. Yaralioglu, O. Oralkan, A. S. Ergun, D.-S. Lin, S. H. Wong, and B. T. Khuri-Yakub, "Finite element modeling and experimental characterization of crosstalk in 1-D CMUT arrays," IEEE Trans. Ultrason. Ferroelectr. Freq. Control, vol. 54, no. 2, pp. 418-430, 2007.

[2] P. C. Eccardt and A. Lohfink, "Analysis of crosstalk between fluid coupled CMUT membranes," in Proc. IEEE Ultrasonics Symp., 2005, pp. 593-596.

[3] X. Jin, F. L. Degertekin, and B. T. Khuri-Yakub, "Characterization of one-dimensional capacitive micromachined ultrasonic immersion transducer arrays," IEEE Trans. Ultrason. Ferroelectr. Freq. Control, vol. 48, no. 3, pp. 750-760, 2001.

[4] C. Audoly, "Some aspects of acoustic interactions in sonar transducer arrays," J. Acoust. Soc. Am., vol. 89, no. 3, pp. 1428-1433, 1991.

[5] D. L. Carson, "Diagnosis and cure of erratic velocity distributions in sonar projector arrays," J. Acoust. Soc. Am., vol. 34, no. 9, pp. 1191-1196, 1962.

[6] C. H. Sherman, "Analysis of acoustic interactions in transducer arrays," IEEE Trans. Sonics Ultrason., vol. 13, no. 1, pp. 9-15, 1966.

[7] A. Caromi, D. Fiasca, G. Caliano, M. Pappalardo, and E. Cianci, "Experimental study of acoustic coupling in CMUT arrays by optical interferometry," in Proc. IEEE Ultrasonics Symp., 2003, pp. 1960-1964.

[8] A. Caronti, A. Savoia, G. Caliano, and M. Pappalardo, "Acoustic coupling in capacitive microfabricated ultrasonic transducers: Modeling and experiments," IEEE Trans. Ultrason. Ferroelectr. Freq. Control, vol. 52, no. 12, pp. 2220-2234, 2005.

[9] K. K. Park, M. Kupnik, H. J. Lee, B. T. Khuri-Yakub, and I. O. Wygant, "Modeling and measuring the effects of mutual impedance on multi-cell CMUT configurations," in Proc. IEEE Ultrasonics Symp., 2010, pp. 431-434.

[10] S. Zhou and J. A. Hossack, "Reducing inter-element acoustic crosstalk in capacitive micromachined ultrasound transducers," IEEE Trans. Ultrason. Ferroelectr. Freq. Control, vol. 54, no. 6, pp. 1217$1228,2007$.

[11] A. Caronti, G. Caliano, R. Carotenuto, A. Savoia, M. Pappalardo, E. Cianci, and V. Foglietti, "Capacitive micromachined ultrasonic transducer (CMUT) arrays for medical imaging," Microelectron. J., vol. 37 , no. 8 , pp. $770-777,2006$.

[12] H. Lee, J. Tak, W. Moon, and G. Lim, "Effects of mutual impedance on the radiation characteristics of transducer arrays," J. Acoust. Soc. Am., vol. 115, no. 2, pp. 666-679, 2004.

[13] J. Lee, I. Seo, and S.-M. Han, "Radiation power estimation for sonar transducer arrays considering acoustic interaction," Sens. Actuators $A$, vol. 90, no. 1-2, pp. 1-6, 2001.

[14] N. M. Senlik, S. Olcum, H. Koymen, and A. Atalar, "Radiation impedance of an array of circular capacitive micromachined ultrasonic transducers," IEEE Trans. Ultrason. Ferroelectr. Freq. Control, vol. 57, no. 4, pp. 969-976, 2010. 
[15] C. H. Sherman and J. L. Butler, Transducers and Arrays for Underwater Sound (The Underwater Acoustics Series). New York, NY: Springer, 2007.

[16] S. Ballandras, M. Wilm, W. Daniau, A. Reinhardt, V. Laude, and R. Armati, "Periodic finite element/boundary element modeling of capacitive micromachined ultrasonic transducers," J. Appl. Phys., vol. 97, no. 3, art. no. 034901, 2005.

[17] G. G. Yaralioglu, A. S. Ergun, and B. T. Khuri-Yakub, "Finite-element analysis of capacitive micromachined ultrasonic transducers," IEEE Trans. Ultrason. Ferroelectr. Freq. Control, vol. 52, no. 12, pp. $2185-2198,2005$.

[18] C. Meynier, F. Teston, and D. Certon, "A multiscale model for array of capacitive micromachined ultrasonic transducers," J. Acoust. Soc. Am., vol. 128, no. 5, pp. 2549-2561, 2010.

[19] H. Köymen, A. Atalar, E. Aydoğdu, C. Kocabas, H. K. Oğuz, S. Olcum, A. Ozgurluk, and A. Ülügedik, "An improved lumped element nonlinear circuit model for a circular CMUT cell," IEEE Trans. Ultrason. Ferroelectr. Freq. Control, vol. 59, no. 8, pp. 17911799, 2012

$20]$ D. T. Porter, "Self- and mutual-radiation impedance and beam patterns for flexural disks in a rigid plane," J. Acoust. Soc. Am., vol. 36, no. 6, pp. 1154-1161, 1964 .

[21] H. K. Oğuz, A. Atalar, and H. Köymen, "Analysis of mutual acoustic coupling in CMUT arrays using an accurate lumped element nonlinear equivalent circuit model," in Proc. IEEE Ultrasonics Symp., 2012

[22] H. Köymen, A. Atalar, and H. K. Oğuz, "Designing circular CMUT cells using CMUT biasing chart," in Proc. IEEE Ultrasonics Symp., 2012.

[23] A. S. Savoia, G. Caliano, and M. Pappalardo, "A CMUT probe for medical ultrasonography: From microfabrication to system integration," IEEE Trans. Ultrason. Ferroelectr. Freq. Control, vol. 59, no. 6, pp. 1127-1138, Jun. 2012

[24] F. Y. Yamaner, S. Olcum, H. K. Oğuz, A. Bozkurt, H. Köymen, and A. Atalar, "High-power CMUTs: Design and experimental verification," IEEE Trans. Ultrason. Ferroelectr. Freq. Control, vol. 59, no. 6, pp. 1276-1284, 2012

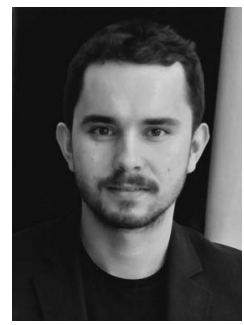

H. Kağan Oğuz was born in Ankara, Turkey in 1985. He received his B.S. and M.S. degrees in electrical engineering in 2006 and 2009, respectively, both from Bilkent University, Ankara, Turkey. Between 2009 and 2012, he worked as an R\& D engineer at Meteksan Defence Industry Inc., Underwater Acoustic Systems Division, Ankara. Since 2009, he has been working toward his Ph.D. degree in the Electrical and Electronics Engineering Department at Bilkent University, where he is currently a research assistant. His current research interests include design and fabrication of underwater transducers and CMUTs.

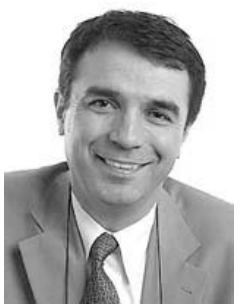

Abdullah Atalar received his B.S. degree from the Middle East Technical University, Ankara, Turkey, in 1974, and M.S. and Ph.D. degrees from Stanford University, Stanford, CA, in 1976 and 1978, respectively, all in electrical engineering. He worked for Hewlett Packard Labs, Palo Alto, in 1979. From 1980 to 1986, he was on the faculty of the Middle East Technical University as an Assistant Professor. In 1986, he joined Bilkent University as the chairman of the Electrical and Electronics Engineering Department and served in the founding of the department, where he is currently a Professor. In 1995 , he was a Visiting Professor at Stanford University. From 1996 to 2010 , he was the Provost of Bilkent University. He is presently the Rector of the same university. His current research interests include micromachined devices and microwave electronics.

Prof. Atalar was awarded the Science Award of TUBITAK in 1994. He is a Fellow of IEEE and a member of Turkish Academy of Sciences.

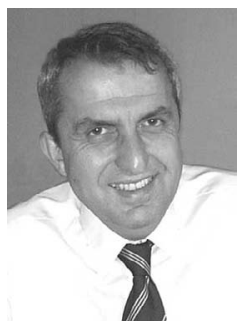

Hayrettin Köymen received the B.Sc. and M. Sc. degrees from the Middle East Technical University (METU), Ankara, Turkey, in 1973 and 1976, respectively, and the Ph.D. degree from Birmingham University, UK, in 1979, all in electrical engineering. He worked as a faculty member in the Marine Sciences Department (Mersin) and Electrical Engineering Department (Ankara) of METU from 1979 to 1990, and at Bilkent University since 1990, where he is a professor. His research activities have included underwater acoustic and ultrasonic transducer design, acoustic microscopy, ultrasonic NDT, biomedical instrumentation, mobile communications, and spectrum management.

Prof. Köymen is a fellow of IET (formerly IEE). 